Vesna Jovanović-Mihaylov

Università della Slesia, Katowice

Polonia

(iD https://orcid.org/0000-0003-0875-0001

\section{Lucyna Marcol-Cacoń}

Università della Slesia, Katowice

Polonia

(iD https://orcid.org/0000-0003-0332-3078
Fraseologismi con la componente somatica cuore nella lingua croata e italiana. Approccio contrastivo

Phraseological units with a somatic component heart

in Croatian and Italian - a comparative study

\begin{abstract}
The article provides a cognitive analysis of phraseological units with the heart component in comparative terms. The purpose of the analysis is to show the similarities and differences in expressing emotions (positive, neutral and negative) between two languages originating from different linguistic groups: the Croatian language (South Slavic group) and the Italian language (from the group of the Romance languages). Phraseological units are analysed on the basis of three criteria: identical in both languages; partially adequate in both languages; idiomatic for one language. The research presents the motivation of phraseologisms and aims to prove that the heart is related to human emotional life and is a container for feelings.
\end{abstract}

Keywords

Phraseologisms, somatism heart, Croatian, Italian, comparative analysis

\title{
1. Introduzione
}

L'oggetto di analisi del presente articolo è la componente somatica cuore presente in alcune espressioni fraseologiche - croate ed italiane - osservata dal punto di vista dell'esternazione delle emozioni positive, neutre e negative. Lo 
studio comparativo nell'ambito della fraseologia di queste due lingue è un tema raramente trattato da parte dei linguisti ed è quindi diventato lo stimolo principale per affrontare tale argomento.

Occorre precisare sin dall'inizio che per i fraseologismi somatici si considerano, dopo Černàk, le espressioni che hanno una parte del corpo umano come una sua componente (Černàk, 1998: 112). Tutti gli aggettivi che si riferiscono al corpo umano vengono dunque chiamati somatici (treccani.it, voce: somatico, accesso: 06/08/2021). È utile aggiungere che nonostante il corpo umano svolga le stesse funzioni a prescindere dalla lingua, le sue estensioni metaforiche possono essere determinate culturalmente (Kovačević, 2012: 16-17).

La fondatezza nel condurre un'analisi contrastiva nell'ambito della fraseologia italiana e croata si trova in stretta correlazione con le ricerche indirizzate all'immagine linguistico-culturale del mondo dei paesi di lingua slava e romanza, il che è stato preso in considerazione nell'interpretazione dei risultati del presente studio (Anusiewicz, 1995: 23).

La scelta del corpus è legata alla considerazione che la capacità di comprendere varie reazioni emotive nonché azioni umane, insieme alla corretta presentazione dei propri giudizi nei contatti con gli utenti stranieri della lingua (nel caso specifico) croata ed italiana, dipende in una certa misura dall'abilità di comprendere determinati fraseologismi e quindi anche dall'atteggiamento nei confronti dei rappresentanti di un'altra cultura.

Dal punto di vista storico, occorre prendere in considerazione il fatto che il territorio dell'attuale Croazia, fino al primo secolo dopo Cristo, fu abitato dai popoli illirici, mentre nei secoli che vanno dal sesto al settimo, dai popoli slavi. Invece, la causa della fuga di parecchi Croati e il conseguente insediamento di una piccola parte di questi nella Penisola Appenninica fu l'aggressione da parte dei Turchi (XV e XVI secolo).

Le cittadine come Acquaviva Collecroce (nome croato-molisano: Živavoda Kruč), Montemitro (nome croato-molisano: Mundimitar) nonché San Felice del Molise (nome croato-molisano: Filić) sono situate nei territori dove gli abitanti parlano fino al giorno di oggi la lingua croata e dove la popolazione croata (2 mila circa) è riuscita a mantenere l'identità nazionale. L'elemento che contraddistingue tale identità è la lingua molisano-croata (K. Feruga, 2008: 26).

Vale la pena sottolineare la presenza degli Slavi sul territorio delle altre regioni del Sud dell'Italia. Nel XI secolo vennero fondate parecchie colonie slave in Puglia. Gli Slavi segnalarono anche la loro presenza in Campania e Calabria. Molte denominazioni geografiche provengono dal periodo delle migrazioni ed anch'esse indicano il legame con gli Slavi. Sul territorio del promontorio Gargano, ad esempio, vi sono cittadine come Punta Crovatico ovvero Grotta Crovatico, mentre nelle altre zone della Puglia si trovano località come Vicinia di Scibinico e Ponte Alma di Trau, le cui denominazioni nacquero per analogia con i nomi delle città croate: Šibenika i Trogiru (J. Vince-Pallua, 1996: 20). Non bisogna sorvolare neanche sul 
fatto che una parte delle odierne regioni croate, vale a dire Dalmazia ed Istria, per molti secoli si trovavano sotto una forte influenza culturale italiana.

\section{Principali aspetti teorici}

Si propone di cominciare con la considerazione di Francesca Casadei che descrive come fraseologismo ogni espressione lessicale satura che fa parte di un discorso ripetuto di una data comunità linguistica a prescindere dalla trasparenza semantica e connotazione pragmatico-comunicativa (F. Casadei, 1995: 355).

Già nella metà degli anni 80 del XX secolo, Bożena Rejakowa descrisse la fraseologia comparativa come "sezione della linguistica comparativa che analizza la struttura formale e la struttura semantica delle sequenze di parole fisse sia dal punto di vista delle somiglianze che dal punto di vista delle divergenze"1 (B. Rejakowa, 1986: 9). All'interno della scuola strutturalista, ci si concentrava sull'analisi delle caratteristiche puramente linguistiche. La linguistica post-strutturale, invece, si basava anzitutto sulla ricerca delle differenze tra le modalità di formazione delle immagini, sull'esperienza nonché sulla descrizione dei frammenti del mondo. I linguisti che si occupano di fraseologia giungono sovente alla considerazione che in questa branca della linguistica vengono osservati riferimenti ai fenomeni linguistici, culturali nonché cognitivi in base ai quali si sono formate all'epoca le espressioni fraseologiche.

A questo proposito è utile menzionare che nella prospettiva della linguistica cognitiva, la lingua è strettamente legata ai processi mentali responsabili della percezione della realtà.

Nell'ambito degli studi cognitivi vi sono parecchie teorie, tra le quali quella dell'immagine linguistica del mondo, che si manifesta anzitutto nel lessico e nella fraseologia, sottoposti al continuo cambiamento (A. Šmelev, 2004). Facendo riferimento alla definizione proposta da Anna Pajdzińska, l'immagine linguistica del mondo "è un insieme di giudizi più o meno fissati nelle strutture della lingua i quali comunicano le caratteristiche ed i modi dell'esistenza della realtà extralinguistica nonché delineano generali categorie concettuali le quali formano il modo di pensare dell'uomo sul mondo"2 (A. Pajdzińska, 1996: 170). Occorre mettere inoltre in evidenza, come già accennato, che l'immagine linguistica del mondo non è uno specchio della realtà, ma è un'interpretazione di tale realtà da parte dei parlanti, la quale si manifesta nella grammatica nonché nel lessico e nella fraseologia (J. Bartmiński, 1990: 10).

\footnotetext{
${ }^{1}$ Traduzione propria.

2 Traduzione propria.
} 
È evidente dunque che le immagini linguistiche di qualsiasi due lingue, anche quelle imparentate, non si sovrappongono reciprocamente in maniera completa (A. Spagińska-Pruszak, 2005: 16). Nelle espressioni fraseologiche si evidenziano le caratteristiche che si possono considerare rilevanti per una data comunità linguistica (J. Anusiewicz, A. Dąbrowska, M. Fleischer, 2000: 34). Le assenze di corrispondenze tra le lingue riguardano in primo luogo i nomi concreti. Quanto ai nomi delle parti del corpo umano, è interessante notare che ruka in croato può significare sia mano che braccio in italiano. Quanto ai fraseologismi somatici, con particolare riguardo a quelli con la componente cuore, le differenze lessicali che si verificano tra il croato e l'italiano, sembrano essere dovute principalmente ai fattori storici e culturali.

La comprensione delle relazioni tra il piano dell'espressione e quello del contenuto di particolari espressioni fraseologiche nella lingua italiana e croata non è affatto facile. Chi accetta tale compito dovrebbe riferirsi alla loro motivazione globale in quanto spiegazione della metafora plasmante un'unità fraseologica (A. M. Lewicki, 2003: 282). La decomposizione del significato di ciascun fraseologismo può essere riconducibile a fattori molto diversi. Nel presente studio verrà sottoposto all'analisi il tipo di motivazione stereotipata, che secondo Andrzej Maria Lewicki, si riferisce ai pregiudizi a proposito di un dato oggetto i quali non risultano dall'osservazione né dalla definizione dell'oggetto, ma dalle convinzioni, dai pregiudizi oppure dalle credenze (A. M. Lewicki, 2003: 286). La motivazione di molti fraseologismi è dovuta ai fattori socio-culturali che svolgono un ruolo principale nella decifrazione del significato metaforico di una data espressione fraseologica. Questo, secondo Lewicki, è il tipo più vicino alla motivazione lessicale per il fatto che i giudizi sui referenti delle componenti formanti una data espressione coesistono nella costruzione del significato di un'intera espressione fraseologica.

Lewicki distingue anche la motivazione simbolica. Per tale motivazione sono contraddistinte le espressioni fraseologiche che sono segni indicanti segni.

I fraseologismi di questo tipo sono anzitutto espressioni (i cosiddetti predicati fraseologici) costruite in base ai comportamenti umani nonché sulle osservazioni (A. M. Lewicki, 2003: 285).

Occorre tener presente che il significato simbolico della componente somatica cuore svolge un ruolo precipuo nella formazione di un dato significato fraseologico insieme ad un'altra componente / altre componenti che sono rappresentate in molti casi dai verbi. Essi intensificano pertanto le emozioni ed evocano connotazioni positive, negative o neutre.

Nell'opinione di Anna Pajdzińska (2003: 75), la motivazione semantica dei fraseologismi è strettamente legata all'immagine linguistica del mondo ossia ad una serie di giudizi più o meno radicati nella lingua che concernono le caratteristiche della realtà extralinguistica. La linguista conferma che la maggior parte 
dei fraseologismi possiede una genesi metaforica ovvero, molto più raramente, metonimica (A. Pajdzińska, 2003: 76).

In molti casi è difficile definire la motivazione di particolari espressioni fraseologiche per il fatto che non è dato per scontato da quali situazioni extralinguistiche e da quale conoscenza sul mondo queste derivino. Bisogna però tentare di dare una risposta alla seguente domanda:

attraverso quali esperienze umane e per via di quali credenze, convinzioni e superstizioni l'immagine del mondo radicata nella lingua croata e italiana potrebbe essersi formata? E quindi come ne può essere scaturito il proprio significato fraseologico?

Secondo Piotr Müldner-Nieckowski (2004: 15), non è facile definire in maniera univoca la genesi di un dato fraseologismo per il fatto che l'origine della maggior parte di essi è sconosciuta. L'autore chiarisce che si può soltanto intuire quali circostanze e quali associazioni hanno portato alla formazione di ogni espressione esaminata nel presente articolo, ma non è possibile stabilire quando, dove e da chi queste espressioni vennero pronunciate per la prima volta. Inoltre, si mette in risalto che non è facile stabilire il percorso dei prestiti di particolari espressioni fraseologiche da diverse lingue, anzi in parecchi casi risulta un'operazione impossibile. Occorre rispondere ai seguenti quesiti: quale lingua adottò una data espressione per prima e da che lingua deriva nonché se all'epoca venne adottato un intero fraseologismo sulla base di elementi di una cultura preesistente. A questo proposito vale la pena fare riferimento all'opinione di Halina Kurkowska e di Stanisław Skorupka, i quali sottolineano che:

Di solito, i ricercatori hanno a che fare con formazioni che insorgono indipendentemente, in maniera parallela in due o più lingue. Tali analogie si possono spiegare con il fatto che sia gli uomini che gli animali, nelle loro reazioni più naturali nonché nel loro comportamento, sono in tutto il mondo uguali (H. Kurkowska, S. Skorupka, 2001: 162)3.

Nonostante vi siano differenze tra gruppi linguistici sull'intero continente europeo, occorre prendere in considerazione il fatto che le espressioni fraseologiche esistenti nelle lingue europee presentano molte caratteristiche comuni risultanti in primo luogo dall’influenza della cultura greco-latina.

\footnotetext{
${ }^{3}$ Traduzione propria.
} 


\section{Basi empiriche della ricerca}

L'analisi confrontativa tra le due lingue si basa sul corpus elaborato in base ai dizionari croati: Anić (1998), Matešić (1982), Menac, Fink-Arsovski, Venturin (2003) ed in base ai dizionari italiani: Zingarelli (2003), Pittano (1994, 1996), Drzymała (1993), Salwa, Szleszyńska (1997), Podracka (2006) ed è stato suddiviso in tre categorie:

1) fraseologismi con la componente cuore di struttura formale identica e significato simile;

2) fraseologismi con la componente cuore di struttura formale parzialmente simile e significato simile;

3) fraseologismi con la componente cuore che differiscono sia nella struttura formale che nel loro significato tra la lingua italiana e quella croata.

Si precisa che nella suddetta tripartizione dei fraseologismi, il criterio distintivo è costituito dalla maggiore o minore somiglianza tra il croato e l'italiano. Lo studio comparativo dei fraseologismi contenenti la parola cuore permette di comprendere meglio l'immagine usata in un dato fraseologismo.

Le espressioni prescelte per il suddetto esame servono anzitutto all'espressione dello stato emotivo dell'emittente e non del destinatario. Le emozioni che vengono comunicate sono positive, neutre ovvero negative. La componente cuore è stata scelta per il significato sia simbolico-connotativo che denotativo di tale voce somatica.

Come afferma Anna Wierzbicka (1971: 8), il cuore "è come se fosse un uomo intero [...]. E come se fosse l'interiorità dell'uomo (tranne la mente)" ${ }^{4}$. Anna Pajdzińska (2003: 95) sottolinea che "dal punto di vista linguistico il cuore non è più l'organo che serve a pompare il sangue nel corpo, ma un elemento al quale la sfera emotiva dell'uomo è strettamente legata" 5 .

L'analisi comparativa comprende 76 espressioni fraseologiche, 34 voci croate e 42 italiane. L'obiettivo del presente studio mira a descrivere delle somiglianze nonché delle divergenze tra le espressioni fraseologiche contenenti la parola cuore nell'ottica cognitivista (in base alla teoria dell'immagine linguistica del mondo) sull'esempio di due lingue provenienti da due gruppi distinti: la lingua croata (gruppo meridionale delle lingue slave) e la lingua italiana (gruppo di lingue romanze).

\footnotetext{
${ }^{4}$ Traduzione propria.

${ }^{5}$ Traduzione propria.
} 


\section{Fraseologismi con la componente cuore di struttura formale identica e significato simile}

I fraseologismi croati ed italiani facenti parte di questo gruppo sono esempio di una corrispondenza totale delle immagini linguistiche del mondo croato e quello italiano. Si sottolinea che sia nella lingua croata che in quella italiana le componenti che costituiscono particolari espressioni fraseologiche presentano una simile struttura formale e un simile significato. Facendo riferimento alla tipologia proposta da Földes (1996), i fraseologismi presentati sotto sono esempio dell'equivalenza totale.

In entrambi le lingue, il cuore è spesso descritto in categorie di esperienza amorosa. Diventa per l'appunto il cuore, in quanto contenitore di emozioni, il fulcro con cui si esprimono l'amore, la soddisfazione o la felicità. Gli esempi riportati di seguito considerano il cuore come simbolo di amore ovvero di affettuosità e hanno un carattere simbolico:

- croato: pokloniti (predavati / predati) <svoje> srce komu // italiano: donare (dare) il cuore a qualcuno;

- croato: otvarati / otvoriti (otkriti, rastvoriti) komu <svoje> srce // italiano: aprire il cuore a qualcuno;

- croato: nositi (imati) koga, što u srcu // italiano: portare qualcuno nel cuore;

- croato: $i z$ (od) <sveg(a)> srca / svim (čitavim, punim) srcem // italiano: con tutto il cuore (di tutto cuore).

Il fraseologismo tuče (puca, kuca) komu srce za kim, za čim (croato) // il cuore batte per qualcuno (italiano) ha un carattere intralinguistico. La frequenza cardiaca viene regolata dal sistema nervoso dell'uomo, mentre il cuore, grazie alla sua funzione fisiologica in combinazione con il verbo battere, implica l'intensità dei sentimenti.

L'espressione slušati glas svojeg srca (croato) // ascoltare la voce del cuore (italiano) suscita connotazioni positive. È probabile che i fattori socio-culturali, uniti a vissuti e ad esperienze personali (vale a dire ascoltare il proprio intuito, ascoltare quello che ci suggerisce il cuore), svolgano un ruolo importante, mentre si prova a decifrare il significato di tale espressione. L'espressione fraseologica imati lavlje srce (croato) // avere il cuore di leone (italiano) offre l'immagine di una persona coraggiosa, disposta a dare una mano ad altre persone. Il leone, secondo la spiegazione proposta nel dizionario dei simboli da Władysław Kopaliński (1990: 193), simboleggia, tra l'altro, forza, vittoria, coraggio, impegno, mobilità. Il leone è anche emblema del cuore e del sangue, mentre il cuore di leone simboleggia la forza nonché la prodezza (W. Kopaliński, 1990: 195).

Il cuore è anche visto come una preda in battaglia: osvojiti / osvajati čije srce // conquistare il cuore di qualcuno. 
Il cuore non è solo il luogo dei sentimenti positivi, ma anche di quelli negativi. Può diventare simbolo di indignazione, irritazione o di rabbia. È anche concettualizzato come un oggetto fragile che può essere facilmente rotto o frantumato: - croato: slomiti / lomiti komu srce // italiano: spezzare il cuore a qualcuno.

Da quest'espressione emerge l'immagine dell'uomo che porta un'altra persona alla disperazione, negando o rifiutando l'amore. Nell'espressione successiva: (croato): raniti koga u srce // (italiano): pugnalare qualcuno dritto al cuore è illustrato lo stato emotivo di una persona triste, afflitta da ferite psicologiche o danni emotivi da parte di altre persone. Il significato di tale espressione sembra risultare dalle osservazioni delle esperienze umane, serve per descrivere il comportamento altrui e per di più, ciascuna componente è usata letteralmente. Il cuore in quanto sede della tristezza, del rimpianto, del dolore fa parte di molte espressioni fraseologiche in ambedue le lingue analizzate, nelle quali crea l'immagine di una persona afflitta che soffre a causa di emozioni negative. Occorre prestare attenzione all'espressione seguente: croato: srce nekome krvari // italiano: far sanguinare il cuore a qualcuno. Il sangue insieme al colore rosso in riferimento alla sfera emotiva dell'uomo simboleggiano anzitutto desiderio e passione (W. Kopaliński, 1990: 162). La perdita di sangue, invece, ha connotazioni negative e significa la perdita di forza ossia di vitalità. Il sangue che fuoriesce dal corpo umano indica una situazione anomala (J.-P. Roux, 1994: 58). I fraseologismi successivi nešto leži komu na srcu (croato) // qualcosa sta a cuore a qualcuno (italiano); srce se steže (steglo) komu (croato) // (si) stringe il cuore a qualcuno (italiano) illustrano lo stato emotivo di un individuo sopraffatto dal dolore. La motivazione delle suddette espressioni ha carattere intralinguistico. Si aggiunga inoltre che entrambe trasmettono una caratteristica negativa.

L'ultima espressione di significato negativo, sulla quale si vuole porre accento nel presente articolo, è la seguente:

- croato: srce udara kao bubanj // italiano: il cuore batte come un tamburo.

Nel caso specifico si tratta del fraseologismo il cui significato è legato con le sensazioni negative come paura, preoccupazione, ansia, tensione. L'espressione presenta una motivazione simbolica. Il tamburo che rulla era all'epoca simbolo ovvero segnale dell'imminente guerra (W. Kopaliński, 1990: 14).

\section{Fraseologismi con la componente cuore aventi struttura formale simile e significato parzialmente simile in italiano e in croato}

Nel presente gruppo sono stati classificati fraseologismi croati ed italiani simili tra loro dal punto di vista del significato. Ciò che li differenzia è anzitutto 
il diverso ordine dei costituenti, ma anche, ad esempio, il ricorso a diverse parti del discorso. Facendo riferimento alla tipologia proposta da Földes (1986), i fraseologismi individuati in questo gruppo sono un esempio di equivalenza parziale. All'interno di questo gruppo vi sono due sottogruppi:

- equivalenza semantica totale con l'equivalenza formale parziale o zero,

- equivalenza semantica parziale o zero con l'equivalenza formale totale o parziale.

I fraseologismi presentati sotto fanno parte del primo sottogruppo, mentre non vi è alcun esempio da poter essere classificato nel secondo sottogruppo.

Si propone di presentare alcuni fraseologismi che si differenziano per l'ordine dei costituenti. Nonostante il significato in ambedue le lingue sia mantenuto, occorre sottolineare che la posizione delle parti del discorso nei fraseologismi inerenti ad ambedue le lingue è diversa. Nella lingua croata vi è l'aggettivo seguito dal sostantivo, mentre in italiano, soprattutto a causa della mancanza di declinazione, nella maggior parte dei fraseologismi, l'aggettivo segue il sostantivo. Tale regola viene confermata da una serie di esempi di carattere metaforico:

- croato: laka srca // italiano: a cuor leggero;

- croato: otvorena srca // italiano: a cuore aperto;

- croato: iz dubine srca (iz dna srca) // italiano: dal profondo del cuore;

- croato: imati zlatno srce // italiano: avere un cuore d'oro;

- croato: imati zečje srce // italiano: avere un cuore di coniglio.

È utile soffermarsi sull'ultima espressione che, al contrario delle quattro precedenti aventi connotazioni positive, è connotata negativamente: imati zečje srce // avere un cuore di coniglio. Nell'arte medievale, il coniglio che inseguiva un cavaliere simboleggiava la vigliaccheria (W. Kopaliński, 1990: 484) e perciò il coniglio è diventato, tra l'altro, simbolo della pusillanimità sia in croato che in italiano.

L'espressione srce je zadrhtjelo komu (croato) // il cuore palpita (a qualcuno) (italiano), che si riferisce a chi ha paura, è usata nella lingua croata con il verbo al tempo passato, mentre in italiano è solita apparire con il verbo al tempo presente. Tale fraseologismo è legato al carattere intralinguistico. L'equivalente croato simile all'espressione soprastante è il seguente: srce komu udara kao malj - il cuore batte a qualcuno come un martello con il significato di avere paura di fare qualcosa ovvero di affrontare una situazione. Si precisa inoltre che tale espressione non è presente nella lingua italiana. Il martello (croato: malj) inteso come un'arma è simbolo, tra l'altro, di potenza, forza, violenza e via discorrendo (W. Kopaliński, 1990: 230). L'espressione crea l'immagine di una persona che va incontro a certe esperienze tutt'altro che positive, il che è legato a forti emozioni - paura, ansia, angoscia. Tali esperienze negative fanno sì che il cuore di chi le sperimenta batte forte, palpita rapidamente.

A proposito dell'espressione italiana il cuore palpita (a qualcuno) vanno fatte alcune osservazioni. Al suo posto viene usata di frequente l'espressione avere le palpitazioni. A volte si sente in alternativa l'espressione il cuore batte come un 
tamburo. Basti ritornare al capitolo precedente per vedere che tale espressione italiana possiede il suo equivalente in croato.

Le espressioni fraseologiche con la componente nominale cuore che evocano connotazioni positive creano l'immagine di una persona soddisfatta e felice. Esso avviene sia nella lingua croata sia in quella italiana, il che è illustrato dall'espressione seguente: puno je komu srce (croato) // sentirsi ridere il cuore oppure il cuore mi sorride (italiano). Al posto dell'avverbio croato puno vi sono i verbi ridere e sorridere in italiano.

L'espressione pao je (spao je) teret (kamen) sa srca komu (croato) / toglier(si) un peso dal cuore (italiano) significa che si prova un grande sollievo. Nella lingua italiana, nel caso specifico appare il sostantivo peso accompagnato dal verbo togliere, che nella lingua croata è sostituito dal verbo cadere - pao. Il fraseologismo possiede un altro equivalente nella lingua croata però con la componente seno - grudi: pao je teret s grudi komu (croato). Il sostantivo teret (sasso) simboleggia difficoltà, durezza, immobilità (W. Kopaliński, 1990: 140). Quando è accompagnato dal verbo cadere, in croato crea l'immagine di una persona che ottiene ovvero prova sollievo nonché si è liberata da preoccupazioni e problemi. Le connotazioni simili evoca il sostantivo peso in ambedue le lingue.

\section{Fraseologismi con la componente cuore, differenti tra lingua croata ed italiana}

Le espressioni con la componente cuore (croato: srce) sono molto produttive nella lingua croata e fra queste vi sono tante voci appartenenti al gruppo medievale. Tali espressioni possono essere usate per descrivere una persona ossia lo stato emotivo di una persona che affronta determinate situazioni che possono essere positive, negative ovvero neutre. I fraseologismi appartenenti al presente gruppo corrispondono, secondo la tipologia di Földes (1996), all'equivalenza zero ossia alla situazione nella quale le unità di partenza non hanno corrispondenti nella lingua di arrivo. La traduzione di tali unità esige da parte di chi la svolge il ricorso a diversi procedimenti quali parafrasi, traduzione tramite spiegazione, aggiunta, descrizione, compensazione e via discorrendo.

Lo stato emozionale positivo dell'uomo viene espresso dai fraseologismi che presentano l'immagine di un uomo soddisfatto e felice. Si vedano le espressioni seguenti: srce raste (zaigra, kliče, igra) komu; srce gori komu; vuče srce koga za čim (čemu). Inoltre, gli ultimi due esempi presentano l'immagine di una persona nella quale si risveglia un desiderio. L'espressione fraseologica olakšalo (odlaknulo) je kоти srcu crea l'immagine di una persona che prova pace e sollievo in seguito alla liberazione da un sentimento spiacevole o da un problema. Le tre 
espressioni esposte di seguito si contraddistinguono dal significato simile all'espressione appena citata. L'immagine è espressa con le estensioni metaforiche seguenti:

- pustiti (dati) srcu na volju;

- istresti (izliti, razgaliti) srce;

- dati / davati (pustiti / puštati) srcu maha.

Nella lingua croata, molte espressioni fraseologiche vengono utilizzate per esprimere emozioni negative. Le più caratteristiche contenenti la componente cuore sono le seguenti: imati zlo srce na koga, iskaliti / iskaljivati srce (žuč) na koga, biti nakraj srca, stajati kao trn pod srcem komu, imati srce u petama.

Il cuore non è quindi solo sede di emozioni positive e neutre, ma anche di quelle connotate negativamente.

Vi è nel croato l'espressione imati zlo srce na koga che si riferisce a qualcuno di un atteggiamento negativo nei confronti di un'altra persona. Le connotazioni negative vengono anche evocate dall'espressione biti nakraj srca, che vuol dire essere arrabbiato con qualcuno senza motivo.

Nella lingua croata vi sono due fraseologismi con i quali si manifesta la paura nonché la rabbia. Il primo contiene la componente srce: iskaliti/iskaljiati srce na koga con il significato di prendersela con qualcuno, mentre il secondo presenta lo stesso significato ma contiene la componente žuč: iskaliti/iskaljivati žuč na koga, dove la bile (croato: $\check{z} u c ̌$ ) simboleggia l'amarezza e il rancore.

Vale la pena soffermarsi sull'espressione fraseologica stajati kao trn pod srcem kоти con il significato di essere ostacolo per qualcuno. La spina (croato: trn) simboleggia, tra l'altro, disagio, difficoltà, ansia e pericolo (W. Kopaliński, 1990: 509).

L'esempio successivo, che evoca emozioni negative, è l'espressione imati srce u petama, il cui significato è quello di 'morire di paura'. A tale espressione si può ricorrere, quando si vuole illustrare una persona che sperimenta determinati eventi o situazioni legati ad uno stress emotivo ed altre emozioni forti come paura, angoscia ed ansia. Tale fraseologismo prende origine da osservazioni nonché da esperienze umane.

La parola italiana cuore, similmente a quanto avviene nella lingua croata, appare in molte espressioni fraseologiche tipiche, il significato delle quali è strettamente legato alla sfera emotiva dell'uomo. I fraseologismi sono legati al significato metaforico. Si può ricorrere a tali espressioni nelle situazioni in cui il parlante vuole esprimere in parole lo stato emotivo positivo in quanto reazione ad una determinata situazione. Alla presente categoria si possono classificare le espressioni seguenti:

- guadagnarsi il cuore di qualcuno,

- rubare il cuore a qualcuno,

- toccare il cuore di qualcuno,

- mettere (anche metterci) il cuore in quello che si fa. 
Oltre a quelli appena elencati, vale la pena menzionare il fraseologismo parlare con il cuore in mano ossia parlare cordialmente, in cui il cuore si incontra con la componente somatica mano. Il significato dell'espressione italiana mettersi una mano sul cuore è quello di ricorrere alla propria coscienza. È utile aggiungere che sia l'espressione cuore in mano che una mano sul cuore si associano alla franchezza, alla rettitudine e alla genuinità (W. Kopaliński, 1990: 350, 372). Un'altra espressione nella quale accanto al cuore appare un'altra parte del corpo ossia le labbra è la seguente: avere il cuore sulle labbra, alla quale si può ricorrere, mentre si vuole sottolineare la franchezza, nonché genuità di qualcuno. Il tratto positivo viene anche espresso dall'espressione avere cuore, che viene molto utilizzata per indicare magnanimità, generosità e bontà. L'espressione italiana il cuore brama si usa in riferimento anzitutto ad una persona che desidera ardentemente qualcuno oppure qualcosa. Inoltre, esiste in italiano l'espressione di gran cuore, che oltre al significato simile all'espressione di tutto il cuore, può essere usata in riferimento ad una persona dal cuore grande.

Tenendo presente che il cuore è spesso descritto come un contenitore nel quale risiedono le emozioni, occorre mettere in risalto che nella lingua italiana viene metaforizzato "in forma liquida" la felicità straripa dal cuore ovvero come gas $i l$ cuore gonfio di gioia.

In forma neutra può essere valutata l'espressione non avere il cuore di fare qualcosa alla quale si ricorre, quando si intende descrivere una persona che non ha coraggio né audacia per fare qualcosa.

È utile analizzare le espressioni nelle quali il cuore diventa sede di emozioni negative: rodersi / mangiarsi il cuore, stare con il cuore in pena, avere il cuore pesante, sentirsi il cuore di piombo.

Si può osservare che a volte, il significato del lessema decide del senso peggiorativo di un dato fraseologismo. Le unità lessicali come pesante, di piombo oppure pena evocano esperienze negative - peso, sofferenza, ansia. Vale la pena menzionare inoltre che oltre all'espressione rodersi il cuore si può incontrare nella lingua italiana il fraseologismo rodersi dalla bile. Il significato di ambedue le espressioni è simile nonostante il fatto che in ciascun fraseologismo dopo lo stesso verbo appare un'altra parte del corpo. Al posto del cuore può apparire bile, che viene associata all'amarezza e alla rabbia.

\section{Conclusioni}

Nel presente contributo è stato condotto uno studio comparativo di alcune espressioni fraseologiche croate ed italiane nelle quali è presente la componente somatica cuore. L'analisi, comprendente 76 espressioni fraseologiche nella lingua 
croata ed italiana, ha rivelato molte somiglianze che risultano prevalentemente dalla provenienza di tutte e due le culture dalla cultura europea e latina nonché ha rivelato anche divergenze tra tali due lingue di origine diversa.

I fraseologismi sono stati raggruppati in tre categorie: fraseologismi identici in ambedue le lingue, quindi tali che coincidono perfettamente dal punto di vista della struttura e del significato; fraseologismi che coincidono parzialmente dal punto di vista della struttura morfo-sintattica ma ciononostante mantengono lo stesso significato; fraseologismi idiomatici della lingua croata e della lingua italiana.

L'espressione delle emozioni nella cultura croata ed italiana è strettamente correlata con il cuore per il fatto che tale organo viene associato prevalentemente con la sfera emotiva dell'uomo. Molte delle somiglianze tra le due lingue messe a confronto sono non soltanto il risultato dell'attingere alle stesse fonti, ma sono anche diretta conseguenza della compartecipazione dei Croati e degli Italiani allo scambio di modelli culturali tra le nazioni europee.

Bisogna però ricordare che nonostante vi siano molte espressioni fraseologiche identiche, ve ne sono anche tante altre che derivano da diverse tradizioni ed esperienze storiche e culturali di una o dell'altra nazione.

A tali differenziazioni tra i fraseologismi nella lingua croata e italiana hanno contribuito le diverse vicende storiche delle due nazioni, le distinte tradizioni culturali nonché le mentalità all'interno delle stesse società, ognuna con i propri sistemi di valori.

\section{Riferimenti bibliografici}

Anić, V. (1998). Rječnik hrvatskoga jezika. Zagreb, Novi Liber.

Anusiewicz, J. (1995). Lingwistyka kulturowa. Zarys problematyki. Wrocław, Wydawnictwo Uniwersytetu Wrocławskiego.

Anusiewicz, J., Dąbrowska, A., \& Fleischer, M. (2000). Językowy obraz świata i kultura. Projekt koncepcji badawczej. Język a kultura, 13, 11-44.

Bartmiński, J. (1990). Językowy obraz świata. Lublin, Wydawnictwo Uniwersytetu Marii Curie-Skłodowskiej.

Bocian, E. (2017). La concettualizzazione metaforica delle emozioni nella lingua italiana. Annales Universitatis Paedagogicae Cracoviensis Studia de Cultura, 9(1), 29-36.

Casadei, F. (1995). Per una definizione di espressione idiomatica e una tipologia dell'idiomatico in italiano. Lingua e stile, $X X X(2), 335-358$.

Čermák, F. (1998). Somatic idioms revisited. In W. Eismann (Ed.), Europhras 95 - Europäische Phraseologie im Vergleich (pp. 109-119). Bochum, Brockmeyer.

Drzymała, P. (1993). Fraseologia italiana. Słowniczek frazeologiczny włosko-polski. Poznań, Wydawnictwo Naukowe UAM. 
Feruga, K. (2008). Socjolingwistyczne uwarunkowania języka molizańskich Chorwatów. http://www.sbc.org.pl/Content/11968/doktorat2828.pdf (accesso: 22/10/2019).

Földes, C. (1996). Deutsche Phraseologie kontrastiv. Intra- und interlinguale Zugänge. Heidelberg, Julius Groos Verlag.

Kopaliński, W. (1990). Stownik symboli. Warszawa, Wiedza Powszechna.

Kovačević, B. (2012). Hrvatski frazemi od glave do pete. Zagreb, Institut za hrvatski jezik i jezikoslovlje.

Kurkowska, H., \& Skorupka, S. (2001). Stylistyka polska. Warszawa, Wydawnictwo Naukowe PWN.

Lewicki, A. M. (2003). Studia z teorii frazeologii. Łask, Oficyna Wydawnicza LEKSEM.

Matešić, J. (1982). Frazeološki rječnik hrvatskoga ili srpskog jezika. Zagreb, Školska knjiga.

Matsumoto, D., \& Juang, L. (2007). Psychologia międzykulturowa. Gdańsk, Wydawnictwo Psychologiczne.

Menac, A., Fink-Arsovski, Ž., \& Venturin, R. (2003). Hrvatski frazeološki rječnik. Zagreb, Naklada Ljevak.

Müldner-Nieckowski, P. (2004). Nowy szkolny słownik frazeologiczny. Warszawa, Bertelsmann Media - Świat Książki.

Pajdzińska, A. (1996). Znaczenie związku frazeologicznego. In Z. Krążyńska \& Z. Zagórski (Red.), Poznańskie Spotkania Językoznawcze, 1 (s. 168-173). Poznań, Wydawnictwo Poznańskiego Towarzystwa Przyjaciół Nauk.

Pajdzińska, A. (2003). Studia frazeologiczne. Lask, Oficyna Wydawnicza LEKSEM.

Pittano, G. (1994). Sinonimi e contrari: dizionario fraseologico delle parole equivalenti, analoghe e contrarie. Bologna, Zanichelli.

Pittano, G. (1996). Frase fatta capo ha: dizionario dei modi di dire, proverbi e locuzioni. Bologna, Zanichelli.

Podracka, M. (2006). Idiomy włoskie. Warszawa, Rea.

Rejakowa, B. (1986). Związi frazeologiczne o identycznej lub podobnej budowie morfologicznej w języku słowackim i polskim. Wrocław, Zakład Narodowy im. Ossolińskich.

Roux, J.-P. (1994). Krew. Mity, symbole, rzeczywistość (M. Perek, Przeł.). Kraków, Znak.

Salwa, P., \& Szleszyńska, M. (1997). Wybór idiomów włoskich. Warszawa, Wiedza Powszechna.

Spagińska-Pruszak, A. (2005). Intelekt we frazeologii polskiej, rosyjskiej i chorwackiej. Z problemów językowego obrazu świata. Łask, Oficyna Wydawnicza LEKSEM.

Šmelev, A. (2004). O slovare ključevych slov russkoj jazykovoj kartiny mira. Russkij Jazyk Segodnja, 4, 347-353.

Vince-Pallua, J. (1996). Doprinos utvrđivanju tragova Hrvata u južnoj Italiji. In H. Salopek (Ed.), Tjedan moliških Hrvata (pp. 18-26). Zagreb, Hrvatska Matica Iseljenika.

Wierzbicka, A. (1971). Kocha, lubi, szanuje. Medytacje semantyczne. Warszawa, Wiedza Powszechna.

Zingarelli, N. (2003). Lo Zingarelli. Vocabolario della lingua italiana. Bologna, Zanichelli. 